\title{
Content-Aware Cooperative Multiple Access Protocol for Packet Speech Communications
}

\author{
Amr El-Sherif ${ }^{1}$, Andres Kwasinski ${ }^{2}$, Ahmed Sadek ${ }^{1}$, and K. J. Ray Liu ${ }^{1}$ \\ ${ }^{1}$ Department of Electrical and Computer Engineering, \\ ${ }^{2}$ Texas Instruments Inc., \\ University of Maryland, College Park, MD 20742, USA. \\ \{aasherif, aksadek, kjrliu\}@umd.edu \\ Germantown, MD 20874, USA. \\ akwasinski@ieee.org
}

\begin{abstract}
A novel cooperative multiple access protocol for packet speech communications is proposed. Cooperation is achieved through a relay node and by exploiting the silence periods during speech communication. The relay forwards packets for the active calls during some of the time slots that are free from those users which are silent. Therefore, no extra channel resources are needed for cooperation and the system encounters no bandwidth losses. A Markov model characterizing the network operation is built and thoroughly analyzed. The throughput and delay performance of the proposed protocol are characterized and compared to a similar non-cooperative protocol for speech communications.
\end{abstract}

\section{INTRODUCTION}

Cooperative communications is a new paradigm for wireless networks where multiple users collaborate by creating multiple signal paths to relay information for each other. These multiple signals are combined at a destination so as to create spatial diversity. As most of the work on cooperative communications has focused on the physical layer, few works have focused on the impact and implementation of cooperation at higher network layers. In [1], the authors proposed a distributed version of the network diversity multiple access (NDMA) protocol [2] and provided analysis to demonstrate the diversity gain. In [3], the authors presented the notion of utilizing the spatial separation between users to assign cooperating pairs. In [4], the authors proposed a cognitive multiple access protocol that enables cooperation by benefiting from data burstiness to let a relay utilize periods of transmission silence from empty data queues. Also [4] studied the maximum stable throughput and delay performance of the cognitive protocol.

Speech networks differs from data networks because of the talk-silence patterns that characterize voice traffic. These patterns have long been exploited in statistical multiplexinglike schemes [5] where users who are silent, release their channel resources, which can then be utilized to admit more users to the network. In this work, this concept is extended by making a relay operating in incremental decode-and-forward mode [6] use some of the released resources to help users in a talk state forward their packets with higher reliability. A new cooperative multiple access protocol is proposed, where the freed up resources by the silent users is intelligently divided between the cooperative relay and new users demanding access to the network.

We study the performance of the proposed protocol by describing its state evolution through a Markov chain. We gain insight into the dynamics of the network and the tradeoffs associated with the presence of the relay. We characterize the throughput and delay performance of our proposed protocol, and compare them with a network that does not implement cooperation. Furthermore, we study the packet dropping probability and, in the application layer, the speech quality. Our numerical results show significant gains of the proposed cooperative protocol over the non-cooperative one.

\section{SYSTEM MODEL}

We consider the uplink channel of a wireless network carrying conversational voice traffic. The network employs a modified version of the packet reservation multiple access (PRMA) protocol as a medium access protocol. PRMA can be viewed as a combination of TDMA and slotted ALOHA protocols where access to the channel is divided into time frames, which are further subdivided into $N$ time slots each.

Speech sources are characterized by periods of silence in between talk spurts that account for roughly $60 \%$ of a conversation time [7]. To model this, each speech source in a conversation is modeled as a Markov chain as shown in Fig. 1(a) with two states: talk (TLK) and silence (SIL) state. The basic time unit for the Markov chain is the duration of one complete frame of length $T$ seconds. This means that state transitions are only allowed at frame boundaries. Then, the transition probability from state TLK to state SIL is the probability that a talk spurt with mean duration $t_{1}$ ends in a frame of duration $T$, that is, $\gamma=1-e^{-T / t_{1}}$. Similarly, for a silence of mean duration $t_{2}$, the transition probability from state SIL to state TLK is $\sigma=1-e^{-T / t_{2}}$.

The on-off nature of speech is used to increase network utilization by granting access to the channel only to users in the TLK state. In PRMA, users starting a talk spurts contend independently for the channel in empty time slots. A slot is reserved for a user that has used it for a successful contention; otherwise, the base station feeds back a NULL message to make the slot available for contention in the next time frame. Here, we assume immediate feedback. The reserved slots are used to transmit voice packets. At the end of a talk spurt, the user enters a silence state where it is not generating or transmitting any packets. In this case, the base station feeds back a NULL message declaring the previously reserved time slot once again free for the use of other calls.

To represent the wireless channel, the signal received at the base station or the relay is modeled as

$$
y_{i}=\sqrt{P_{1} r_{i}^{-\alpha}} h_{i} x+\eta_{i}
$$

where $i \in\{B, R\}$ is the base station or the relay index, $x$ is the transmitted signal, $P_{1}$ is the transmission power, assumed to 


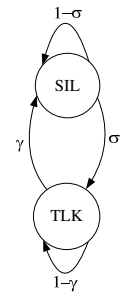

Fig. 1. System models (a) Speech source model, (b) User's terminal model

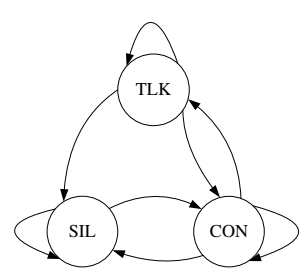

Fig. 1. System models (a) Speech source model, (b) User's terminal model

be the same for all terminals, $r_{i}$ denotes the distance between a node and its destination, $\alpha$ is the path loss exponent, $h_{i}$ the channel fading coefficients, modeled as zero-mean complex Gaussian random variables with unit variance, and $\eta_{i}$ is an additive noise term, modeled as zero-mean complex Gaussian random variable with variance $N_{0}$. We assume that the channel coefficients are constant for the duration of the transmission of one packet. In this work, we only considered the case of a symmetric network, where all the inter-users channels are assumed to be statistically identical.

We characterize the success and failure of packet reception by outage events and outage probabilities. The outage probability is defined as the probability that the Signal to Noise Ratio (SNR) at the receiver is less than a given SNR threshold $\beta$, called outage $S N R$ [8]. For the channel model in (1) the received $\mathrm{SNR}$ is

$$
\mathrm{SNR}_{i}=\frac{\left|h_{i}\right|^{2} r_{i}^{-\alpha} P_{1}}{N_{0}}
$$

where $\left|h_{i}\right|^{2}$ has an exponential distribution with unit mean. Accordingly, the probability of outage is given by,

$$
P_{O i}=\operatorname{Pr}\left\{\left|h_{i}\right|^{2}<\frac{\beta N_{0} r_{i}^{\alpha}}{P_{1}}\right\}=1-\exp \left(-\frac{\beta N_{0} r_{i}^{\alpha}}{P_{1}}\right) \text {. }
$$

\section{Proposed Cooperative Protocol}

Transmission errors in the wireless channels have a significant impact on performance [9]. On one side, a user will fail a contention for a time slot and will have to contend again in another slot if it experiences a channel error. Moreover, a user holding a reserved slot has to give it up and go through the contention process again because after a channel error the ensuing NULL feedback from the base station indicates that the slot is free. Both effects translate into an increase in contending users and, thus, a significant increase in network traffic and in delay to gain a slot reservation, which ultimately severely degrades the speech quality. In fact, the congestion may reach a level where all users experience reduced speech quality due to packets dropped due to excessive delay [5].

In a voice network, one can mitigate the wireless channel impairments through the spatial diversity offered by cooperation. We propose the deployment of a single relay node, which helps users holding slot reservations, forward their packets through an incremental decode-and-forward (DF) protocol. This means that, upon a request from the base station, the relay forwards the packets that were not successfully received by the base station but were successfully decoded by the relay. This increases the reliability in the terminal-base station link and, hence, reduces the number of users loosing their reserved time slots. This reduces the average number of contending users, and, therefore, improves speech quality by significantly lowering access delay and packet dropping probability.

To organize the time slots in a frame, we propose a structure where the first $N_{T}$ slots form a variable size (from frame to frame) compartment reserved for the talking users. Of the remaining $\left(N-N_{T}\right)$ slots, a fraction $p_{R}$ is assigned to the relay and the remaining are made available for contention. In the frame, the slots used for contention follow those reserved for talking users and precede the last $N_{R}$ slots, which are assigned to the relay. When a user gives up or gains a slot reservation, the slots are rearranged so as to maintain this frame structure. In any frame, the number of slots assigned to the relay will be $N_{R}=\operatorname{round}\left(p_{R}\left(N-N_{T}\right)\right)$. The value of $p_{R}$ establishes a tradeoff between the amount of help the relay offers and the reduction in the number of time slots available for contention.

\section{Analytical Model}

Based on the system model discussed above, a user can be in one of three states; silence, contending for channel access, or having a reserved slot. The user evolution between these states can be described by the Markov chain of Fig. 1(b). The states, $\{\mathrm{SIL}, \mathrm{CON}$, and TLK $\}$, correspond to the silence, contending and reserved states, respectively. A user in SIL state moves to CON state when a talk spurt begins. A user in CON state will send its older packet when there is an available slot with probability $p_{v}$. If contention succeeds, a user in CON state transits to TLK state where it will have a slot reserved for use in subsequent frames. A user moves from CON state to SIL state if its talk spurt ends before gaining access to the channel. A user in TLK state transits to SIL state when its talk spurt ends, and transits to CON state if its packet is not received correctly by the base station (unless the relay was able to help, in which case it will remain in the TLK state).

Although the actions of different users are independent, the transition probabilities between different states for a given user are in general dependent on the number of users in $\mathrm{CON}$ and TLK states because they will affect the probability of a successful contention. Moreover, the number of users in TLK state will determine the number of slots assigned to the relay, and hence the relay's ability to help users. In order to take these dependencies into consideration, the network will be modeled as the two-dimensional Markov chain $\left(M_{C}, M_{T}\right)$, where $M_{C}$ and $M_{T}$ are random variables denoting the number of users in CON and TLK states, respectively. Assuming there are $M_{v}$ users in the network, the number of users in the SIL state is $M_{S}=M_{v}-M_{C}-M_{T}$. Next, we will analyze this Markov chain so as to derive different performance measures for the cooperative protocol.

Let $S_{1}=\left(M_{C_{1}}, M_{T_{1}}\right)$, and $S_{2}=\left(M_{C_{2}}, M_{T_{2}}\right)$ be the system states at two consecutive frames. Then,

$$
\begin{aligned}
M_{C_{2}} & =M_{C_{1}}+m_{S C}+m_{T C}-m_{C S}-m_{C T} \\
M_{T_{2}} & =M_{T_{1}}+m_{C T}-m_{T S}-m_{T C}
\end{aligned}
$$

where $m_{i j}$ denotes the number of users departing from state $i \in\{S, C, T\}$ to state $j \in\{S, C, T\}$. This implies that 
the transition probability between any two states can be determined from the distributions of $m_{S C}, m_{C S}, m_{C T}, m_{T S}$, and $m_{T C}$, which we derive next.

From Fig. 1(a), and since all users are independent, the number of users transitioning from the SIL to the CON state, $m_{S C}$, follows a binomial distribution with parameter $\sigma$. Then,

$\operatorname{Pr}\left(m_{S C}=i\right)=\left(\begin{array}{c}M_{S} \\ i\end{array}\right) \sigma^{i}(1-\sigma)^{M_{S}-i}, \quad i=0, \ldots, M_{S}$

Upon a successful contention, a user transits from the CON to the TLK state. This transition occurs at the end of each free slot where contention can take place, thus the number of contending users will vary from slot to slot. Suppose there are $M_{T}$ reserved slots and $M_{R}$ relay slots in a given frame, then there are $\left(N-M_{T}-M_{R}\right)$ slots for contention. We want to calculate the distribution of the number of users that moved from CON state to TLK state at the end of the last free slot. This can be calculated using a recurrence model [10]. Let $q\left(M_{C}^{\prime}\right)$ be the probability that a user succeeds in contention when there are $M_{C}^{\prime}$ contending users, then the probability that only one user has permission to transmit and the channel was not in outage during packet transmission is $q\left(M_{C}^{\prime}\right)=M_{C}^{\prime} p_{v}\left(1-p_{v}\right)^{M_{C}^{\prime}-1}\left(1-P_{O B}\right)$. Define $R_{k}\left(M_{C}^{\prime}\right)$ as the probability that $M_{C}^{\prime}$ terminals are remaining in the CON state at the end of the $k t h$ available slot, $(k=0,1,2, \ldots, N-$ $\left.M_{T}-M_{R}\right)$, conditioning on the outcome of the $(k-1)$ st time slot. It follows that for $M_{C}^{\prime}=0,1, \ldots, M_{C}$

$$
\begin{aligned}
R_{k}\left(M_{C}^{\prime}\right)= & R_{k-1}\left(M_{C}^{\prime}\right)\left[1-q\left(M_{C}^{\prime}\right)\right] \\
& +R_{k-1}\left(M_{C}^{\prime}+1\right) q\left(M_{C}^{\prime}+1\right)
\end{aligned}
$$

where $M_{C}$ is the number of users in the CON state at the beginning of the frame. The initial condition for this recursion is $R_{0}\left(M_{C}^{\prime}\right)=1\left\{M_{C}^{\prime}=M_{C}\right\}$, where $1\{\cdot\}$ is the indicator function, and the boundary condition is $q\left(M_{C}+1\right)=0$, which follows from the total number of contending users being $M_{C}$. Finally, the distribution of $m_{C T}$ is

$$
\operatorname{Pr}\left(m_{C T}=i\right)=R_{N-M_{T}-M_{R}}\left(M_{C}-i\right)
$$

From Fig. 1(a) and from users independence, the number users making a transition from the TLK state to the SIL state, $m_{T S}$, is binomially distributed with parameter $\gamma$. Then,

$$
\operatorname{Pr}\left(m_{T S}=i\right)=\left(\begin{array}{c}
M_{T} \\
i
\end{array}\right) \gamma^{i}(1-\gamma)^{M_{T}-i}
$$

A user makes a transition from the CON state to the SIL state if its talk spurt ends before gaining access to the channel. Conditioning on $m_{C T}$, and using the same argument as for $m_{T S}$ above, we have

$$
\operatorname{Pr}\left(m_{C S}=i \mid m_{C T}\right)=\left(\begin{array}{c}
M_{C}^{\prime} \\
i
\end{array}\right) \gamma^{i}(1-\gamma)^{M_{C}^{\prime}-i}
$$

where $M_{C}^{\prime}=M_{C}-m_{C T}$. We will keep this distribution conditioned on $m_{C T}$, because this is the form we will be interested in when calculating the state transition matrix.

A user leaves the TLK state to the CON state if its transmitted packet fails to reach the base station successfully, and the relay did not help that user. Also, a user in TLK state will leave to SIL state if its talk spurt ends in the current frame irrespective of the reception state of its last transmitted packet.
Given $m_{T S}$, the number of transmission errors from users remaining in the TLK state, $\varepsilon$, follows a binomial distribution with parameter $P_{O B}$, the outage probability of the user-to-base station link. Then

$$
\operatorname{Pr}\left(\varepsilon=i \mid m_{T S}\right)=\left(\begin{array}{c}
M_{T}^{\prime} \\
i
\end{array}\right) P_{O B}^{i}\left(1-P_{O B}\right)^{M_{T}^{\prime}-i}
$$

where $M_{T}^{\prime}=M_{T}-m_{T S}$ is the number of users remaining in the TLK state. Assume that the relay can successfully receive $\varepsilon_{R}$ of the $\varepsilon$ erroneous packets. Then, conditioned on $\varepsilon, \varepsilon_{R}$ is also binomially distributed but with parameter $P_{O R}$, the outage probability of the user-to-relay link,

$$
\operatorname{Pr}\left(\varepsilon_{R}=i \mid \varepsilon\right)=\left(\begin{array}{c}
\varepsilon \\
i
\end{array}\right)\left(1-P_{O R}\right)^{i} P_{O R}^{\varepsilon-i}, \quad i=0, \ldots, \varepsilon
$$

For each of the slots assigned to the relay, the relay picks one of the $\varepsilon_{R}$ packets in its queue to forward. It follows that the number of successfully forwarded packets $\varepsilon_{F}$ is binomially distributed with parameter $P_{O B}$,

$$
\operatorname{Pr}\left(\varepsilon_{F}=i \mid \varepsilon_{R}\right)=\left(\begin{array}{c}
M_{R}^{\prime} \\
i
\end{array}\right)\left(1-P_{O B}\right)^{i} P_{O B}^{M-i}
$$

where $M_{R}$ is the number of time slots assigned to the relay and $M_{R}^{\prime}=\min \left(M_{R}, \varepsilon_{R}\right)$ because the number of forwarded packets cannot exceed the number of assigned slots or the number of packets in the relay's queue. Now, the probability that $i$ users make the transition from TLK to CON state is the probability that the relay successfully forwards $\left(\varepsilon_{F}=\varepsilon_{R}-i\right)$ packets out of the $\varepsilon$ erroneous ones. Then,

$$
\operatorname{Pr}\left(m_{T C}=i \mid m_{T S}\right)=\sum_{\substack{k=0\\}}^{\times \operatorname{Pr}\left(\varepsilon_{R}=l \mid \varepsilon=k\right) \operatorname{Pr}\left(\varepsilon=k \mid m_{T S}\right)}
$$

This conditional distribution is the one of interest to calculate the state transition matrix.

Remark: All the distributions calculated above are statedependent because they generally depend on $M_{C}$ and $M_{T}$. This means that we have to calculate a different set of distributions for each possible state of the system.

We can now derive the state transition matrix $\mathbf{P}$. An entry $P\left(S_{1}, S_{2}\right)$ of this matrix is the transition probability from state $S_{1}=\left(M_{C_{1}}, M_{T_{1}}\right)$ to state $S_{2}=\left(M_{C_{2}}, M_{T_{2}}\right)$. If $M_{T_{2}}>$ $\min \left(M_{T_{1}}+M_{C_{1}}, N\right)$, then $P\left(S_{1}, S_{2}\right)=0$ because the number of terminals in TLK state in the next frame cannot exceed the total number of time slots in a frame or the number of terminals in TLK and CON states in the current frame. From (4), (5), and the distributions developed above, the transition probability $P\left(S_{1}, S_{2}\right)$ is given by

$$
\begin{aligned}
& P\left(S_{1}, S_{2}\right)=\sum_{x=0}^{M_{C_{1}}} \sum_{y=0}^{M^{\prime}} \sum_{z=0}^{M_{T_{1}}} \operatorname{Pr}\left(m_{C S}=x \mid m_{C T}=y, S_{1}\right) \\
& \quad \times \operatorname{Pr}\left(m_{T C}=z \mid m_{T S}=M_{T_{1}}-M_{T_{2}}+y-z, S_{1}\right) \\
& \quad \times \operatorname{Pr}\left(m_{S C}=M_{C_{2}}-M_{C_{1}}+x+y-z \mid S_{1}\right) \\
& \quad \times \operatorname{Pr}\left(m_{T S}=M_{T_{1}}-M_{T_{2}}+y-z \mid S_{1}\right) \\
& \quad \times \operatorname{Pr}\left(m_{C T}=y \mid S_{1}\right)
\end{aligned}
$$

where $M^{\prime}=\min \left(M_{C_{1}}-x, N-M_{T_{1}}-M_{R_{1}}\right)$. Note that $\operatorname{Pr}\left(m_{T S}=M_{T_{1}}-M_{T_{2}}+y-z \mid S_{1}\right)=0$ if $M_{T_{1}}-M_{T_{2}}+y-z>$ $M_{T_{1}}-z$, since the number of users transitioning from TLK 
state to CON state cannot exceed the difference between the number of users initially in the TLK state and the number of users leaving the TLK state to the SIL state. Also, $\operatorname{Pr}\left(m_{S C}=\right.$ $\left.M_{C_{2}}-M_{C_{1}}+x+y-z \mid S_{1}\right)=0$ if $M_{C_{2}}-M_{C_{1}}+x+y-z>$ $M_{S_{1}}$, since the number of users leaving the SIL state cannot be larger than the initial number of users in this state.

\section{PERFormanCE ANALYSis}

We consider three performance figures of merit: network throughput, multiple access delay and subjective voice quality. The throughput is the aggregate average amount of data transported through the channel in a unit time. In our case, the number of packets successfully transmitted in a given frame can be decomposed into two components: one originated from successful user contending for channel access and another from the talking users who successfully transmit their packets to the base station (either by themselves or with the help of the relay). Thus, the throughput can be expressed as

$$
T h=\frac{E\left\{E\left\{m_{C T} \mid S_{1}\right\}+M_{T}-E\left\{m_{T C} \mid S_{1}\right\}\right\}}{N}
$$

where $E\{\cdot\}$ is the expectation operator. The outer expectation is with respect to the stationary distribution of the system's Markov chain. The number of successfully transmitted packets is expressed as $M_{T}-E\left\{m_{T C} \mid S_{1}\right\}$, the number of users in TLK state minus the expected number of users leaving the TLK state to the CON state, which are the users with failures in their transmissions.

The multiple access delay is the number of frames a user stays in the CON state before gaining access to the channel. This delay is a function of the probability of a successful contention for a user in a given frame, which depends on the network state at the instant the user enters the CON state, and will change from frame to frame according to the path the network follows in the state space. Then, for exact evaluation of this delay, one should condition on the state at which a user of interest enters the CON state for the first time. Starting from this state, the delay is obtained from the calculation of the statistics of all possible paths the network follows in the state space till the user succeeds in the contention process.

One can show that this method for calculating the delay is impractical. To overcome this difficulty, we approximate the delay as a geometric random variable with parameter $P_{s}(i)$, the contention success probability at state $i$. Then, the approximate average delay is given by $D_{a v g}=\sum_{i \in \Omega} \pi(i) / P_{s}(i)$ where $\Omega$ is the set of states where $M_{C} \neq 0$, and $\pi(i)$ is the ith element of the stationary distribution vector $\pi$. In a given frame, assuming that all user's channels are statistically identical, $P_{s}(i)$ is equal to the probability that at least one user succeeds in contention in that frame, which can be easily computed using the recursion of (7).

We will base the subjective voice quality evaluation of our protocol on the predictive model developed in [11]. This model uses source codec parameters, end-to-end delay and packet dropping probability to predict the value of the Mean Opinion Score $\left(M O S_{c}\right)$ [12] (a subjective voice quality measure that ranges from 1 to 5 ).
Because voice communication is delay sensitive, the network drops all packets with delay exceeding $D_{\max }$ frames. Assuming that the voice coder generates one packet per frame, every user maintains a buffer of length $D_{\max }$. Whenever the buffer is full, the user drops the oldest packet until it succeeds in gaining access to the channel. If the talk spurt ends before reserving a slot, all the packets in the buffer are dropped. Further discussion of this issue is omitted due to space constraints.

\section{NumERICAL RESUlts AND DisCUSSIONS}

We compare the performance of our cooperative multiple access protocol and the PRMA protocol without cooperation. We set each frame with $N=10$ time slots, contention permission probability $p_{v}=0.3$, the SNR threshold $\beta=15$ $\mathrm{dB}$ and the path loss exponent $\alpha=3.7$. The distance between any user and the base station is $100 \mathrm{~m}$, and between any user and the relay $50 \mathrm{~m}$. Speech has a mean talk spurt duration of $t_{1}=1 \mathrm{~s}$. and mean silence period duration of $t_{2}=1.35 \mathrm{~s}$, the maximum delay is $D_{\max }=2$ frames.

Fig. 2 depicts the different performance measures vs. transmission power for $M_{v}=25$ users and for $p_{r}=0.1,0.3$ and 0.5 (amounts of free resources allocated to the relay). Fig. 2(a) depicts the throughput. It is clear that the cooperative protocol outperforms the non-cooperative one. For example, at a power level of $100 \mathrm{~mW}$ and $p_{r}=0.3$, the cooperative protocol shows a $130 \%$ increase in throughput. Note that increasing the amount of resources allocated to the relay increases the gain in throughput, which is expected since more allocated resources means the relay can help more users. Approximate delay is depicted in Fig. 2(b). As the power increases, the delay starts to decrease; then, it increases again. This behavior is because at low power levels the outage probability will be high and even if a user succeeds in contention its packet will be lost with high probability. In this case, increasing the power will decrease the outage probability and hence decrease the delay. It is in this region also that the relay has a positive effect on the delay performance as seen for power levels less than $100 \mathrm{~mW}$. Naturally, a relay is not necessary in any network where terminals are not constrained in transmit power. As power continues to increase, the outage probability will be almost negligible and the main cause of delay will be packet collisions. This, together with the fact that the relay decreases the number of free time slots available for contention, explains why at high power levels the cooperative protocol exhibits larger delay. This is also why the delay performance is better for lower values of $p_{r}$. Finally, Fig. 2(c) shows the estimated conversational Mean Opinion Score. Since this measure depends on both delay and packet dropping probability, the cooperative protocol has better performance at low power level. We can also see that the cooperative protocol provides savings of up to $20 \%$ in power for a good speech quality $(\mathrm{MOSc}=3)$.

We can now draw the following conclusions. At low power levels, the performance of the network is limited by the channel outage event, this is the case when the cooperative protocol outperforms the non-cooperative one thanks to the 

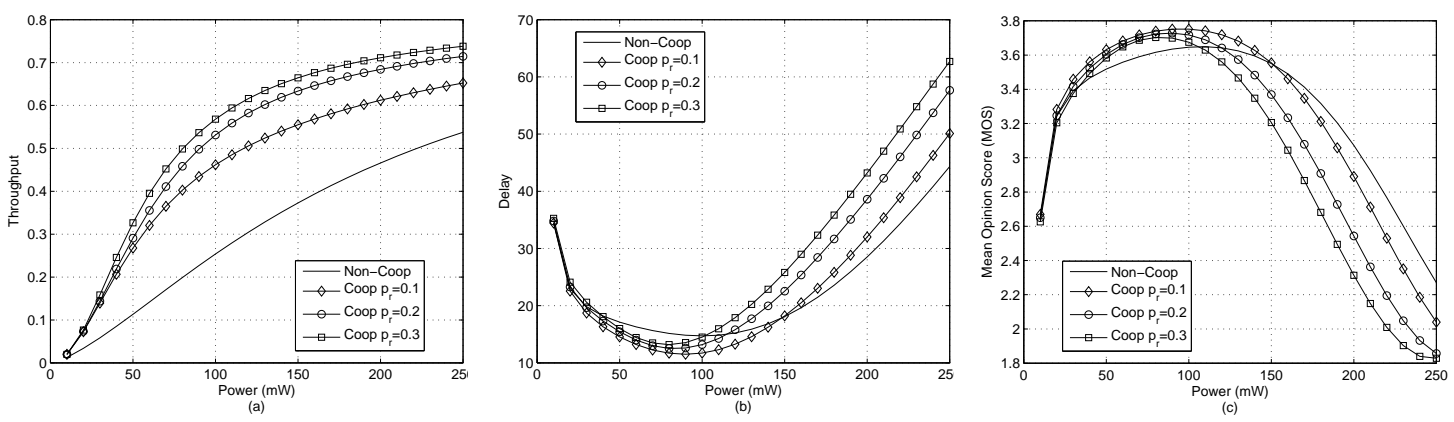

Fig. 2. Network performance measures vs. transmission power for 25 users, (a) Throughput, (b) Delay, and (c) Subjective speech quality.
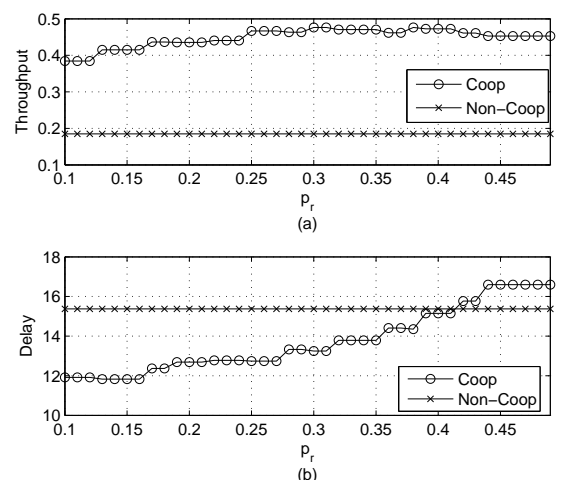

Fig. 3. Network performance measures vs. $p_{r}$ for $75 \mathrm{~mW}$ transmission power level and 25 users, (a) Throughput, (b) Delay

spatial diversity introduced by the relay. On the other hand, for high power levels, the performance is limited by packet collisions. We notice an increase in the average number of reserved slots, because users are less likely to lose their reservations. Therefore, the number of free slots decreases and there is an increase in delay at this region. Since the relay is already using part of the time slots available for contention, the probability of collision increases, and the performance of the cooperative protocol degrades in this region. From Fig. 2(a) and 2(b) one can conclude that, the more the assigned resources to the relay the better the throughput is, because the relay can help more users. On the other hand, delay performance will depend on the amount of transmission power alongside with $p_{r}$, thus, intelligent choice of $p_{r}$ will always guarantee better performance for our protocol. This result is confirmed in Fig. 3 depicting the variation of throughput and delay as a function of $p_{r}$ compared with the non-cooperative case for a network with 25 users and transmission power $P=75 \mathrm{~mW}$. Therefore, the introduction of cooperation in the network forms a tradeoff between the amount of help the relay is offering to the existing users in the network, and the network's ability to accept new users. From Fig. 3 we can see that by assigning between $25 \%$ and $30 \%$ of the free resources to the relay the throughput is almost maximized, furthermore, our protocol outperforms the non-cooperative one in terms of delay.

\section{CONCLUSIONS}

We have proposed a novel multiple access protocol for packet voice communication over wireless relay networks. The relay exploits free resources in the network to intro- duce spatial diversity and help calls forward their packets. We developed and analyzed a Markov model describing the network dynamics in the presence of a relay. We characterized the throughput of the proposed protocol and compared it to the non-cooperative PRMA protocol. Moreover, we studied the delay and subjective speech quality performance of the proposed protocol. Our results shows significant performance gains of the proposed protocol over the non-cooperative counterpart. Furthermore, we studied the tradeoff between the amount of help provided by the relay and the network's ability to accept new users. This tradeoff is due to the relay's occupying a portion of the free resources already used for contention. We showed that the proposed cooperative protocol still outperforms the non-cooperative one in all performance considered measures by intelligently choosing the proportion of resources assigned to the relay.

\section{REFERENCES}

[1] R. Lin and A. P. Petropulu, "Cooperative transmission for random access wireless networks," in Proc. Intl. Conf. on Acoust. Speech and Sig. Proc., Philadelphia, PA, USA, March 2005, pp. 19-23.

[2] M. K. Tsatsanis, R. Zhang, and S. Banerjee, "Network-assisted diversity for random access wireless networks," IEEE Trans. on Signal Processing, vol. 48, pp. 702-711, March 2000.

[3] G. Scutari, S. Barbarossa, and D. Ludovici, "Cooperation diversity in multihop wireless networks using opportunistic driven multiple access," in Proc. of the IEEE Int. Workshop on Signal Processing Advances for Wireless Communications, Rome, Italy, June 2003, pp. 170-174.

[4] A. K. Sadek, K. J. R. Liu, and A. Epheremides, "Collaborative multipleaccess for wireless networks: Protocols design and stability analysis," in Proc. IEEE CISS, Princeton, NJ, 2006.

[5] D. Goodman and S. X. Wei, "Efficiency of packet reservation multiple access," IEEE Trans. on Veh. Tech., vol. 40, no. 1, pp. 170-176, Feb. 1991.

[6] J. N. Laneman, D. N. C. Tse, and G. W. Wornell, "Cooperative diversity in wireless networks: efficient protocols and outage behavior," IEEE Trans. Inform. Theory, vol. 50, pp. 3062-3080, Dec. 2004.

[7] P. T. Brady, "A model for on-off speech patterns in two-way conversation," vol. 48, pp. 2445-2472, Sept. 1969.

[8] N. Benvenuto, S. Pupolin, and G. Guidotti, "Performance evaluation of multiple access spread spectrum systems in the presence of interference," IEEE Trans. on Veh. Tech., vol. 37, no. 2, pp. 73-77, 1988.

[9] M. M. Khan and D. Goodman, "Effects of channel impairments on packet reservation multiple access," in Proc. 44th IEEE Veh. Tech. Conf., Stockholm, Sweeden, June 1994, pp. 1218-1222.

[10] G. Wu, K. Mukumoto, and A. Fukuda, "Analysis of an integrated voice and data transmission system using packet reservation multiple access," IEEE Trans. on Veh. Technology, vol. 43, no. 2, pp. 289-297, May 1994.

[11] L. Sun and E. C. Ifeachor, "Voice quality prediction models and their application in voip networks," IEEE Trans. on Multimedia, vol. 8, no. 4, pp. 809-820, Aug. 2006.

[12] International Telecommunication Union, Methods for Subjective Determination of Transmission Quality, 1996. 